\title{
Bacterial Findings in Infected Hip Joint Replacements in Patients with Rheumatoid Arthritis and Osteoarthritis: A Study of 318 Revisions for Infection Reported to the Norwegian Arthroplasty Register
}

\author{
J. C. Schrama, ${ }^{1}$ O. Lutro, ${ }^{2}$ H. Langvatn, ${ }^{1}$ G. Hallan, ${ }^{1}$ B. Espehaug, ${ }^{3}$ H. Sjursen, ${ }^{2,4}$ \\ L. B. Engesaeter, 1, 3,4 and B.-T. Fevang ${ }^{3,5}$ \\ ${ }^{1}$ Department of Orthopaedics, Haukeland University Hospital, 5021 Bergen, Norway \\ ${ }^{2}$ Section of Infectious Diseases, Medical Department, Haukeland University Hospital, 5021 Bergen, Norway \\ ${ }^{3}$ Norwegian Arthroplasty Register, Haukeland University Hospital, 5009 Bergen, Norway \\ ${ }^{4}$ University of Bergen, Bergen, Norway \\ ${ }^{5}$ Department of Rheumatology, Haukeland University Hospital, 5021 Bergen, Norway
}

Correspondence should be addressed to J. C. Schrama, jcschrama@gmail.com

Received 18 July 2012; Accepted 19 September 2012

Academic Editors: L. Jeys and H. R. Song

Copyright (c) 2012 J. C. Schrama et al. This is an open access article distributed under the Creative Commons Attribution License, which permits unrestricted use, distribution, and reproduction in any medium, provided the original work is properly cited.

\begin{abstract}
High rates of Staphylococcus aureus are reported in prosthetic joint infection (PJI) in rheumatoid arthritis (RA). RA patients are considered to have a high risk of infection with bacteria of potentially oral or dental origin. One thousand four hundred forty-three revisions for infection were reported to the Norwegian Arthroplasty Register (NAR) from 1987 to 2007. For this study 269 infection episodes in 255 OA patients served as control group. In the NAR we identified 49 infection episodes in 37 RA patients from 1987 to 2009. The RA patients were, on average, 10 years younger than the OA patients and there were more females (70\% versus $54 \%)$. We found no differences in the bacterial findings in RA and OA. A tendency towards a higher frequency of Staphylococcus aureus (18\% versus $11 \%$ ) causing PJI was found in the RA patients compared to OA. There were no bacteria of potential odontogenic origin found in the RA patients, while we found $4 \%$ in OA. The bacteria identified in revisions for infection in THRs in patients with RA did not significantly differ from those in OA. Bacteria of oral or dental origin were not found in infected hip joint replacements in RA.
\end{abstract}

\section{Introduction}

Patients with rheumatoid arthritis (RA) often undergo joint surgery, especially prosthetic joint replacements. In the prebiological agent era (before 2000), 1-2\% of patients with RA were estimated to need at least one major joint replacement per year followup [1-4], that is, $25 \%$ of the RA patients with 16-20 years of observation $[5,6]$.

Prosthetic joint infection (PJI) is a serious although infrequent complication to joint replacement surgery. In primary total hip replacements (THR) the risk of deep infection is around $1 \%[7,8]$. A recent study from the Norwegian Arthroplasty Register (NAR) showed that RA patients had the same overall risk of PJI as patients with osteoarthritis in THR, while the risk of revision for infection more than 6 years postoperatively was higher in RA compared to OA patients [9].

In the present paper we seek to evaluate and compare bacterial findings in prosthetic hip joint infections in RA patients versus OA patients, for the following reasons. Firstly, PJIs in patients with RA have been reported to be caused by Staphylococcus aureus (S. aureus) in as much as $37 \%$ [10]. This could be a result of relatively high carriage rates of $S$. aureus in RA patients [11-13]. If this is the case, eradication of nasal S. aureus with intranasal mupirocin ointment perioperatively might offer an attractive 
opportunity for prevention of postoperative prosthetic joint infections caused by $S$. aureus in RA patients undergoing total hip replacement surgery [14-16].

Secondly, RA patients with indwelling hip- or knee-joint prostheses are in some international guidelines considered as exceptional high-risk patients for infections caused by bacteria of potential dental or oral origin. These patients are recommended antibiotic prophylaxis to prevent PJI following bacteremia caused by dental procedures [17-23]. Other guidelines and more recent literature do not mention RA as a high-risk factor and thus do not recommend prophylaxis [24-29] (Table 1). The aims of this study were to evaluate the bacterial findings in PJI among RA patients and compare them to the findings in OA patients with PJI. We particularly focused on the frequency of $S$. aureus. Furthermore, we compared the incidence of PJI caused by microorganisms potentially of oral or dental origin between the groups. This information might contribute in the discussion as to the need for treating the RA patient group different from those with $\mathrm{OA}$, concerning antibiotic prophylaxis.

\section{Material and Methods}

The Norwegian Arthroplasty Register includes information on patient identification, the operating hospital, the reason for and the type of primary and revision operations as well as details on the implant type, the fixation method, and the use of antibiotic prophylaxis in each individual case $[30,31]$. Primarily included in the present study were all patients having had a PJI leading to a revision, (i.e., surgical exchange or removal of parts of or the whole prosthesis) in the period September 15, 1987 until October 2007. The diagnosis PJI was made by the operating surgeon(s) based on clinical judgement of the pre- and peroperative findings at time of revision surgery, since the registry forms are filled in immediately after surgery, and thus before the analysis of bacterial cultures are finished. During the study period 107,535 primary total hip replacements were registered. One thousand four hundred forty-three revision procedures for infection were reported to the NAR. The ten hospitals performing most revisions for infection, with a total of 730 revisions, were visited (during the year 2009) by one of the authors (H.L). In 228 (mean age at revision: 70 years, $56 \%$ females) of the infection episodes the medical records ( $n=36)$, bacterial tests $(n=96)$, or the surgical data were incomplete and/or missing $(n=96)$. Thus, bacterial test reports and the surgeons' description of the revision procedure for a total of 502 episodes were systematically reviewed (Figure 1). In 215 infection episodes the diagnosis, that is, indication for primary surgery, was other than OA or RA, and the major diagnostic groups included acute fracture, sequelae after dysplasia, sequelae after fractura colli femoris, M. Pertes/epiphysiolysis, caput necrosis, and unknown diagnosis. 269 infection episodes were seen in 255 patients with $\mathrm{OA}$ and in the present study these served as a control group.

In the above mentioned cohort 18 infection episodes (14 patients) were observed in patients with RA as the indication for primary surgery. In the complete NAR database another

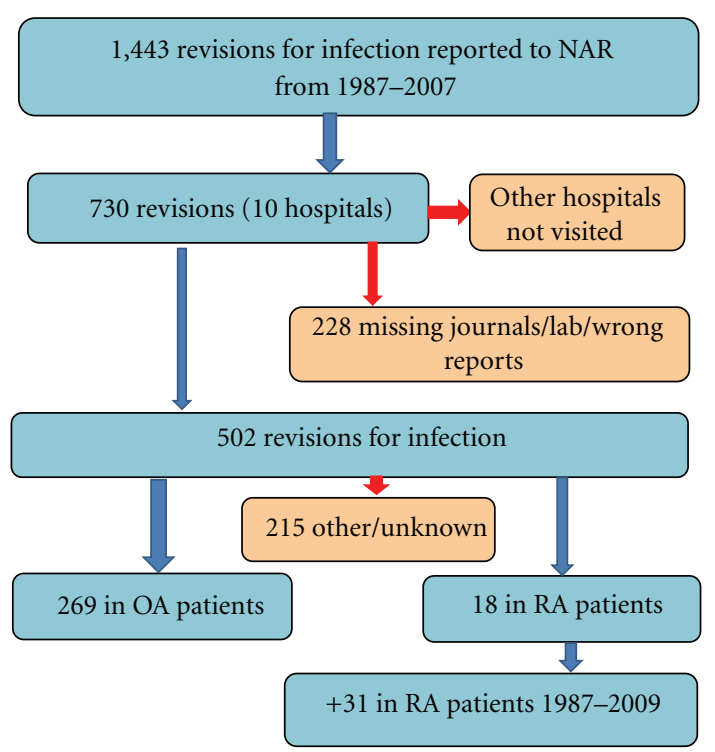

FIGURE 1: Flow chart showing the inclusion of patients for the study.

23 RA patients that had been revised at 10 hospitals not originally visited, were found and their records were obtained. The hospital with most patients was visited by one of the authors (J. C. Schrama) and the other hospitals were contacted by mail and asked to submit a copy of the medical records. A total of 49 infection episodes in 37 RA patients were thus finally included (Figure 1). Included in our analyses were $292 \mathrm{OA}$ and RA patients (mean age at revision: 72 years, $56 \%$ females).

The bacterial findings were obtained from the microbiologic reports in the patient records. Negative cultures (deep and/or biopsy) taken during revision surgery were included and one or more positive cultures were considered as causative for the PJI. We also included bacterial cultures from joint aspiration or blood cultures on the day of revision or 1-2 days before revision surgery. Superficial cultures such as wound swab specimen or swabs from fistulae were excluded. An infection episode (i.e., revision for infection) was considered as a new episode if the patient was assumed clinically free of the former infection and showed unexpected new symptoms of a PJI. Polymicrobial infections, here considered as a separate entity, were defined as infections in which at least two different microorganisms were found. We did not have access to the clinical presentation of the infections, thus no distinction between potentially causative organisms and organisms representing secondary colonisation, could be made. Viridans group streptococci, beta-haemolytic streptococci, Peptostreptococcus species and streptococcus-like organisms not further identified, were considered microbes of potential oral or dental origin, as previously described by Berbari et al. [24]. We defined late infections as infections (i.e., revisions for infection) occurring more than 3 months after implantation surgery according to the definition given by Little et al. [32] and Fitzgerald et al. [33]. 
TABle 1: Overview of literature after year 2000 discussing whether rheumatoid arthritis patients are high risk patients for bacteremic prosthetic joint infection after dental treatment and therefore routinely needing antibiotic prophylaxis.

\begin{tabular}{lccc}
\hline Authors & Year of publication & Country & RA high risk patients, thus antibiotics \\
\hline ADA and AAOS [17] & 2003 & USA & Yes \\
Scott et al. [21] & 2005 & Australia & Yes \\
Tong and Theis [22] & 2008 & New Zealand & Yes \\
Kotze [18] & 2008 & South Africa & Yes \\
Rompen et al. [20] & 2008 & The Netherlands & Yes \\
AAOS [23] & 2009 & USA & Yes \\
Kuong et al. [19] & 2009 & Hong Kong & Yes \\
Seymour et al. [27] & 2003 & Great Britain & No \\
Uçkay et al. [29] & 2008 & Switzerland & No \\
Blomgren et al. [25, 26] & Sweden & No \\
Berbari et al. [24] & 2009 & USA & No \\
\hline
\end{tabular}

\section{Statistics}

Patient characteristics in the RA and OA group were compared using the chi-square test for categorical variables and the student $t$-test for continuous variables. The proportion of a specific microbe in the RA and the OA group (versus the proportion of all other cases) were compared using the chisquare and the Fisher's exact test. Furthermore, multinomial logistic regression (results not shown in table) was used to investigate the relationship between primary diagnosis and bacterial findings. Results were calculated as odds ratios (OR) with 95\% CI comparing the groups CoNS, gram negative bacteria, miscellaneous, mixed flora, and no growth to $S$. aureus. Since a total of 26 patients were registered with more than one revision in the same hip, analyses were also performed based on the first revision only.

Statistical significance was defined as a $P$ value less than 0.05 . Preceding power analysis showed that, based on Berbari's 10 findings of $37 \%$ frequency of S. aureus in RA patients our number of observations would achieve $82 \%$ power to reveal as statistically significant a $20 \%$ difference in group proportions.

\section{Results}

Seventy per cent of patients with RA were females versus $54 \%$ of OA patients $(P=0.06$, Table 2$)$. At the time of revision RA patients were on average 10 years younger than OA patients $(P<0.001$, Table 2$)$. The mean time interval from primary surgery until revision for infection was 3.8 years for RA patients and 3.1 years for OA patients $(P=0.3$, Table 2$)$. Staphylococcus aureus was cultured in 9 of the 49 infection episodes (18\%) in RA patients and 30 of 269 episodes (11\%) in OA patients $(P=0.16$, Table 3$)$. CoNS tended to be a more frequent finding in patients with $\mathrm{OA}$ than in those with RA, although the difference was not statistically significant (18\% RA versus $29 \%$ OA, $P=0.11$, Table 3 ). Using multinomial logistic regression, the odds for culturing CoNS compared to $S$. aureus in RA patients was lower than for OA patients (OR $=0.4,95 \%$ CI: $0.1-1.0, P=0.06)$ indicating that there were more $S$. aureus compared to CoNS in the RA group. Including only the first revision for infection,
TABle 2: Patient characteristics.

\begin{tabular}{lccc}
\hline & RA & OA & $P$ \\
\hline Mean age (years) (SD)* & $64(16)$ & $74(8)$ & $<0.001$ \\
Sex (females\%) ${ }^{* *}$ & $70 \%$ & $54 \%$ & 0.06 \\
Mean time to revision (years) ${ }^{*}$ & 3.79 & 3.13 & 0.3 \\
\hline
\end{tabular}

* In 318 infection episodes, $* *$ in the 292 patients.

TABLE 3: Distribution of cultured bacteria at revision surgery in infected THR in RA patients versus OA patients.

\begin{tabular}{|c|c|c|c|}
\hline & RA $(n=49)$ & OA $(n=269)$ & $P^{*}$ \\
\hline Staphylococcus aureus & $9(18 \%)$ & $30(11 \%)$ & 0.16 \\
\hline $\begin{array}{l}\text { Coagulase negative } \\
\text { staphylococci }\end{array}$ & $9(18 \%)$ & $79(29 \%)$ & 0.11 \\
\hline Streptococci & $1(2 \%)$ & $19(7 \%)$ & 0.33 \\
\hline Enterococci & $1(2 \%)$ & $18(7 \%)$ & 0.33 \\
\hline Gram negative bacteria & $3(6 \%)$ & $10(3 \%)$ & 0.43 \\
\hline Others & $1(2 \%)$ & $10(3 \%)$ & 1.00 \\
\hline Polymicrobial flora & $7(14 \%)$ & $20(7 \%)$ & 0.11 \\
\hline No growth & $18(37 \%)$ & $83(31 \%)$ & 0.42 \\
\hline $\begin{array}{l}\text { Bacteria potentially of oral } \\
\text { or dental origin }\end{array}$ & 0 & $12(4 \%)$ & 0.13 \\
\hline
\end{tabular}

${ }^{*}$ Chi-square and Fisher's exact test.

the difference was statistically significant $(\mathrm{OR}=0.3,95 \% \mathrm{CI}$ : $0.1-0.9, P=0.03)$. Streptococci were cultured in $19(7 \%)$ of the OA patients and in $1(2 \%)$ RA patient $(P=0.33)$. We found no statistically significant difference between the two patient groups with respect to gram negatives $(P=$ $0.43)$, enterococci $(P=0.33)$ and other bacteria $(P=$ 1.00) (Table 3). There was however a tendency towards more polymicrobial cultures in the RA group (14 versus 7\%, $P=$ 0.11 ). Nor was there any statistically significant difference in the frequency of infections in which no bacteria were detected in the culture $(P=0.42)$. Causative microbes, potentially of oral or dental origin, were found in $12(4 \%)$ of the OA and in none of the RA patients $(P=0.13$, Table 3$)$. 


\section{Discussion}

We found no statistically significant differences in the bacterial findings of infected THRs in RA compared to OA patients. Staphylococci were found in more than half $(59.5 \%)$ of the positive cultures as reported by others $[7,34]$.

Mixed or polymicrobial infections had, however, a tendency to be more frequent in the RA group. This finding is in agreement with previous knowledge of wound infections in immune-altered hosts, in whom polymicrobial micro flora is more frequently seen, as for example, in patients with diabetes mellitus [35]. Furthermore, our finding of a high percentage of culture negative infections $(31-37 \%)$ are caused by prior courses of antimicrobial therapy, inappropriate (handling of the) samples or wrong diagnosis.

Another finding in the present study was a tendency towards a higher frequency of $S$. aureus than, for example, CoNS in RA patients compared to OA patients, although the percentage of infections caused by $S$. aureus was not as high as $37 \%$, reported by Berbari and coworkers, but in their study knee as well as hip replacements were included [10]. In the NAR we have no data on revisions for (early) infections in which no implant parts were exchanged or removed. Thus we may have missed some early infections which are frequently caused by virulent bacteria such as $S$. aureus.

Eight of the nine $S$. aureus infections (analyses not shown) found in the RA patients in the present study were late infections, that is, revised more than 3 months postoperatively. RA patients have previously been shown to be more prone to late infections $[9,36,37]$. These late, potentially blood-borne infections have, according to Maderazo et al. [38], skin and soft tissue as the most common remote sites of infection. S. aureus is generally considered unlikely to originate from the mouth and were consequently not included in the group of bacteria of potentially oral or dental origin, in our study. Several authors however advocate the possibility of $S$. aureus originating from the mouth $[39,40]$. Particularly acute or chronic dental infections might increase the possibility of culturing Staphylococci species [39].

In the present study we found no significant difference in the occurrence of microbes potentially of oral or dental origin in RA patients compared with OA patients, and the numerical difference between the groups favoured patients with RA among whom no patient had such a microorganism cultured (as opposed to 12 OA patients). Consequently, our findings do not support guidelines that RA patients are high risk patients particularly vulnerable to PJIs caused by microorganisms after transient bacteremia during dental procedures. The findings are in agreement with the existing policy in Norway which has been that RA patients with THR are not given prophylactic antibiotics before dental treatment.

A strength of this study is that it includes data selected from a national registry comprising an entire country (4.8 million inhabitants) over a period of more than 20 years. Data completeness for hip replacements has been shown to be very good, even for revision operations [41]. Although a large RA cohort has been studied previously (200 infection episodes, Berbari et al. [10]), our material is unique in terms of the comparison of microbiology in one of the largest cohorts of RA and OA patients. A drawback is the insufficient statistical power of the study. A $P$ value in the nonsignificant range can either reflect an actual lack of difference between the patient groups or that there are too few observations to demonstrate such a difference, if existent. Reported findings should be interpreted with this in mind. Our power calculation was based on the findings of Berbari et al. [10] In their material of 200 infection episodes from the prebiological agents era $37 \%$ S. aureus was seen in PJIs in patients with RA. We found only $18 \% S$. aureus in our material and consequently, there were too few infection episodes in our RA patients to detect a statistically significant difference for $S$. aureus (if present). On the other hand our material is, to our knowledge, one of the largest microbiological materials including and comparing RA and OA patients. Another drawback is the large number of infection episodes with missing or wrong data (228 of 730). Although not analysed, we have no reason to believe that these exclusions represent any kind of selection bias.

Furthermore, patients with PJIs treated solely by conservative means or those treated with limited surgical procedures (not involving removal or exchange of prosthetic parts) were not reported to the registry, and thus were not evaluated in this study.

Finally, we had no information on the patients' medication, which might have had an influence on the microbiology. For instance, immune-modulating antirheumatic medication may increase the risk of infection caused by lowvirulent microbes.

\section{Conclusion}

We found no differences in the microbiology of infected THRs in RA patients compared to OA patients. There tended to be an increased risk of PJIs caused by $S$. aureus in RA patients, but we did not confirm the high rates of $S$. aureus previously reported in RA. Whether or not there is reason to advise the use of intranasal mupirocine ointment perioperatively as prophylactic strategy against $S$. aureus in PJI's may still be a matter of discussion, but we found no reason to treat the RA group differently in this respect. Furthermore, RA patients seemed less, rather than more, prone to PJIs caused by potentially oral or dental microbes when compared to OA patients. Consequently we cannot, on the basis of our findings, recommend a different policy regarding antibiotic prophylaxis prior to dental treatment in RA patients. RA patients should be individually evaluated with particular emphasis on the patient's comorbidities and medication [25-27]. We advise to continue the common praxis in Norway not giving routinely antibiotic prophylaxis before dental procedures.

\section{Ethical Approval}

The study with patient identifiable data was approved by the Regional Committee for Medical Research Ethics, Oslo, Norway (number 2009/856b). 


\section{References}

[1] P. Gordon, J. West, H. Jones, and T. Gibson, "A 10 year prospective followup of patients with rheumatoid arthritis 1986-96," Journal of Rheumatology, vol. 28, no. 11, pp. 2409 2415, 2001.

[2] D. James, A. Young, E. Kulinskaya et al., "Orthopaedic intervention in early rheumatoid arthritis. Occurrence and predictive factors in an inception cohort of 1064 patients followed for 5 years," Rheumatology, vol. 43, no. 3, pp. 369376, 2004

[3] T. M. Palm, K. Kaarela, M. S. Hakala, H. J. Kautiainen, H. P. J. Kröger, and E. A. Belt, "Need and sequence of large joint replacements in rheumatoid arthritis. A 25-year follow-up study," Clinical and Experimental Rheumatology, vol. 20, no. 3, pp. 392-394, 2002.

[4] S. M. M. Verstappen, J. N. Hoes, E. J. Ter Borg et al., "Joint surgery in the Utrecht Rheumatoid Arthritis Cohort: the effect of treatment strategy," Annals of the Rheumatic Diseases, vol. 65, no. 11, pp. 1506-1511, 2006.

[5] M. C. Kapetanovic, E. Lindqvist, T. Saxne, and K. Eberhardt, "Orthopaedic surgery in patients with rheumatoid arthritis over 20 years: prevalence and predictive factors of large joint replacement," Annals of the Rheumatic Diseases, vol. 67, no. 10, pp. 1412-1416, 2008.

[6] F. Wolfe and S. H. Zwillich, "The long-term outcomes of rheumatoid arthritis: a 23-year prospective, longitudinal study of total joint replacement and its predictors in 1, 600 patients with rheumatoid arthritis," Arthritis and Rheumatism, vol. 41, pp. 1072-1082, 1998.

[7] J. L. Del Pozo and R. Patel, "Infection associated with prosthetic joints," The New England Journal of Medicine, vol. 361, no. 8, pp. 787-794, 2009.

[8] W. Zimmerli, A. Trampuz, and P. E. Ochsner, "Current concepts: prosthetic-joint infections," The New England Journal of Medicine, vol. 351, no. 16, pp. 1645-1654, 2004.

[9] J. C. Schrama, B. Espehaug, G. Hallan et al., "Risk of revision for infection in primary total hip and knee arthroplasty in patients with rheumatoid arthritis compared with osteoarthritis: a prospective, population-based study on 108,786 hip and knee joint arthroplasties from the Norwegian arthroplasty register," Arthritis Care and Research, vol. 62, no. 4, pp. 473479,2010

[10] E. F. Berbari, D. R. Osmon, M. C. T. Duffy et al., "Outcome of prosthetic joint infection in patients with rheumatoid arthritis: the impact of medical and surgical therapy in 200 episodes," Clinical Infectious Diseases, vol. 42, no. 2, pp. 216223, 2006

[11] M. S. Jackson, J. Bagg, M. N. Gupta, and R. D. Sturrock, "Oral carriage of staphylococci in patients with rheumatoid arthritis," Rheumatology, vol. 38, no. 6, pp. 572-575, 1999.

[12] J. J. Jacobson, B. Patel, G. Asher, J. O. Woolliscroft, and D. Schaberg, "Oral staphylococcus in older subjects with rheumatoid arthritis," Journal of the American Geriatrics Society, vol. 45, no. 5, pp. 590-593, 1997.

[13] D. Tabarya and W. L. Hoffman, "Staphylococcus aureus nasal carriage in rheumatoid arthritis: antibody response to toxic shock syndrome toxin-1," Annals of the Rheumatic Diseases, vol. 55, no. 11, pp. 823-828, 1996.

[14] L. G. M. Bode, J. A. J. W. Kluytmans, H. F. L. Wertheim et al., "Preventing surgical-site infections in nasal carriers of Staphylococcus aureus," The New England Journal of Medicine, vol. 362, no. 1, pp. 9-17, 2010.
[15] D. M. Hacek, W. J. Robb, S. M. Paule, J. C. Kudrna, V. P. Stamos, and L. R. Peterson, "Staphylococcus aureus nasal decolonization in joint replacement surgery reduces infection," Clinical Orthopaedics and Related Research, vol. 466, no. 6, pp. 1349-1355, 2008.

[16] N. Rao, B. Cannella, L. S. Crossett, A. J. Yates Jr., and R. McGough III, "A preoperative decolonization protocol for Staphylococcus aureus prevents orthopaedic infections," Clinical Orthopaedics and Related Research, vol. 466, no. 6, pp. 1343-1348, 2008.

[17] "Antibiotic prophylaxis for dental patients with total joint replacements," Journal of the American Dental Association, vol. 134, no. 7, pp. 895-899, 2003.

[18] M. J. Kotze, "Antibiotic prophylaxis for preventing endocarditis and infection in joint prosthesis after dental treatment: a review of new trends and recommendations in the literature," Journal of the South African Dental Association, vol. 63, no. 8, pp. 440-444, 2008.

[19] E. E. Kuong, F. Y. Ng, C. H. Yan, C. X. S. Fang, and P. K. Y. Chiu, "Antibiotic prophylaxis after total joint replacements," Hong Kong Medical Journal, vol. 15, no. 6, pp. 458-462, 2009.

[20] J. C. Rompen, J. C. M. Schrier, G. H. I. M. Walenkamp, and C. C. P. M. Verheyen, "Indications for antibiotic prophylaxis in patients with a prosthetic joint," Nederlands Tijdschrift voor Geneeskunde, vol. 152, no. 42, pp. 2282-2286, 2008.

[21] J. F. Scott, D. Morgan, M. Avent, S. Graves, and A. N. Goss, "Patients with artificial joints: do they need antibiotic cover for dental treatment?" Australian Dental Journal, vol. 50, no. 4, supplement 2, pp. S45-S53, 2005.

[22] D. C. Tong and J. C. Theis, "Antibiotic prophylaxis and invasive dental treatment in prosthetic joint patients," New Zealand Medical Journal, vol. 121, no. 1280, pp. 45-52, 2008.

[23] http://www.aaos.org/about/papers/advistmt/1033.asp.

[24] E. F. Berbari, D. R. Osmon, A. Carr et al., "Dental procedures as risk factors for prosthetic hip or knee infection: a hospitalbased prospective case-control study," Clinical Infectious Diseases, vol. 50, no. 1, pp. 8-16, 2010.

[25] J. Blomgren, G. Dahlen, U. Dohnhammar, A. Heimdahl, and J. Struwe, "Få indikasjoner for antibiotikaprofylax," Tandläkartidningen, vol. 101, pp. 50-54, 2009.

[26] J. Blomgren, A. Heimdahl, and J. Struwe, "Antibiotic prophylaxis seldom indicated in dental care: important that physicians and dentists agree," Läkartidningen, vol. 106, no. 52, pp. 3485-3486, 2009.

[27] R. A. Seymour, J. M. Whitworth, and M. Martin, "Antibiotic prophylaxis for patients with joint prostheses—still a dilemma for dental practitioners," British Dental Journal, vol. 194, no. 12, pp. 649-653, 2003.

[28] N. A. Simmons, A. P. Ball, R. A. Cawson et al., "Case against antibiotic prophylaxis for dental treatment of patients with joint prostheses," The Lancet, vol. 339, no. 8788, p. 301, 1992.

[29] I. Uçkay, D. Pittet, L. Bernard, D. Lew, A. Perrier, and R. Peter, "Antibiotic prophylaxis before invasive dental procedures in patients with arthroplasties of the hip and knee," Journal of Bone and Joint Surgery B, vol. 90, no. 7, pp. 833-838, 2008.

[30] L. B. Engesaeter, L. I. Havelin, B. Espehaug, and S. E. Vollset, "Artificial hip joints in Norway. A national registry of total hip arthroplasties," Tidsskrift for den Norske Laegeforening, vol. 112, no. 7, pp. 872-875, 1992.

[31] L. I. Havelin, B. Espehaug, S. E. Vollset, L. B. Engesaeter, and N. Langeland, "The Norwegian arthroplasty register: a survey of 17,444 hip replacements 1987-1990," Acta Orthopaedica Scandinavica, vol. 64, no. 3, pp. 245-251, 1993. 
[32] J. W. Little, "The need for antibiotic coverage for dental treatment of patients with joint replacements," Oral Surgery Oral Medicine and Oral Pathology, vol. 55, no. 1, pp. 20-23, 1983.

[33] R. H. Fitzgerald Jr., D. R. Nolan, D. M. Ilstrup, R. E. Van Scoy, J. A. Washington II, and M. B. Coventry, "Deep wound sepsis following total hip arthroplasty," The Journal of Bone and Joint Surgery A, vol. 59, no. 7, pp. 847-855, 1977.

[34] P. C. Matthews, A. R. Berendt, M. A. McNally, and I. Byren, "Diagnosis and management of prosthetic joint infection," British Medical Journal, vol. 338, Article ID b1773, 2009.

[35] P. G. Bowler, B. I. Duerden, and D. G. Armstrong, "Wound microbiology and associated approaches to wound management," Clinical Microbiology Reviews, vol. 14, no. 2, pp. 244269, 2001.

[36] D. A. P. Ainscow and R. A. Denham, "The risk of haematogenous infection in total joint replacements," Journal of Bone and Joint Surgery B, vol. 66, no. 4, pp. 580-582, 1984.

[37] F. E. Stinchfield, L. U. Bigliani, and H. C. Neu, "Late hematogenous infection of total joint replacement," Journal of Bone and Joint Surgery A, vol. 62, no. 8, pp. 1345-1350, 1980.

[38] E. G. Maderazo, S. Judson, and H. Pasternak, "Late infections of total joint prostheses. A review and recommendations for prevention," Clinical Orthopaedics and Related Research, no. 229, pp. 131-142, 1988.

[39] A. H. Friedlander, "Oral cavity staphylococci are a potential source of prosthetic joint infection," Clinical Infectious Diseases, vol. 50, no. 12, pp. 1682-1683, 2010.

[40] I. Olsen, F. Snorrason, and E. Lingaas, "Should patients with hip joint prosthesis receive antibiotic prophylaxis before dental treatment?" Journal of Oral Microbiology, vol. 2, article 5265, 2010.

[41] B. Espehaug, O. Furnes, L. I. Havelin, L. B. Engesæter, S. E. Vollset, and O. Kindseth, "Registration completeness in the Norwegian Arthroplasty Register," Acta Orthopaedica, vol. 77, no. 1, pp. 49-56, 2006. 


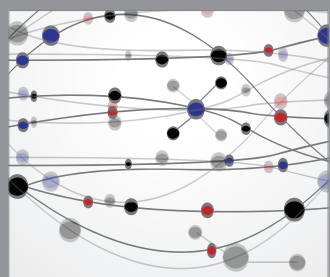

The Scientific World Journal
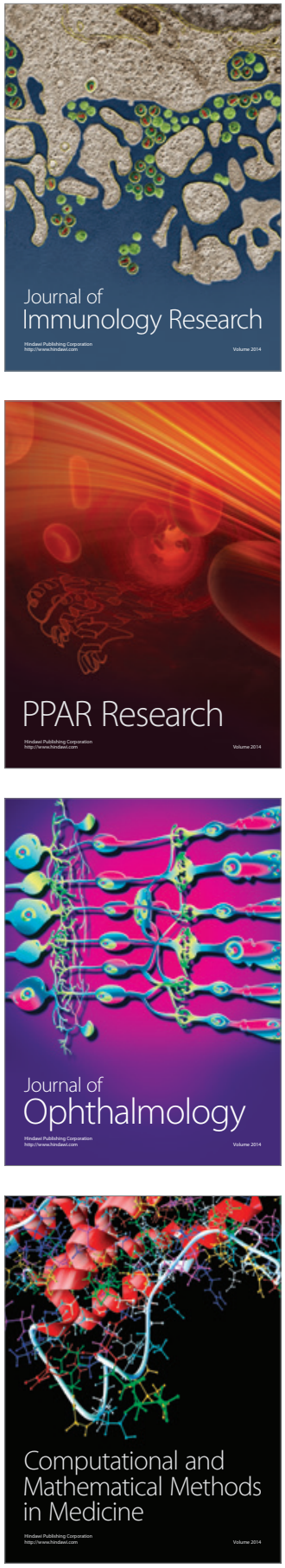

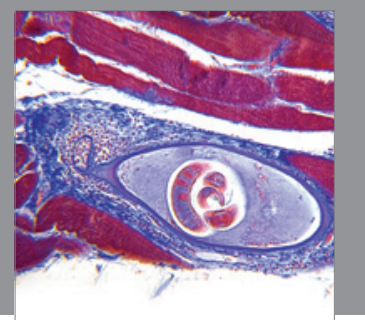

Gastroenterology

Research and Practice
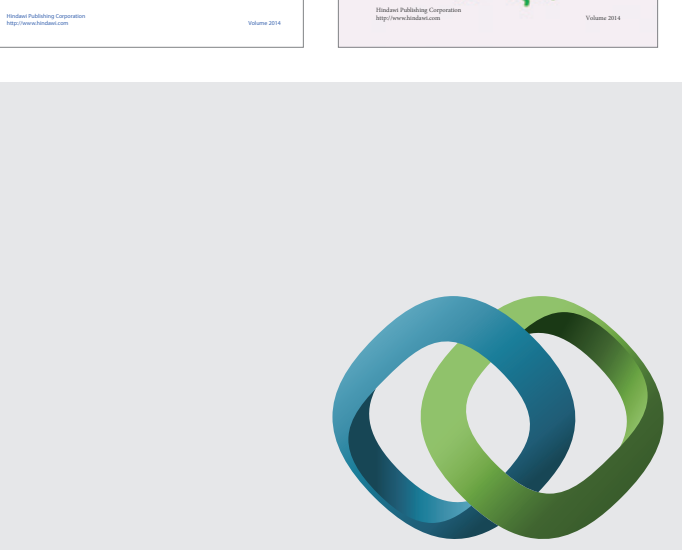

\section{Hindawi}

Submit your manuscripts at

http://www.hindawi.com
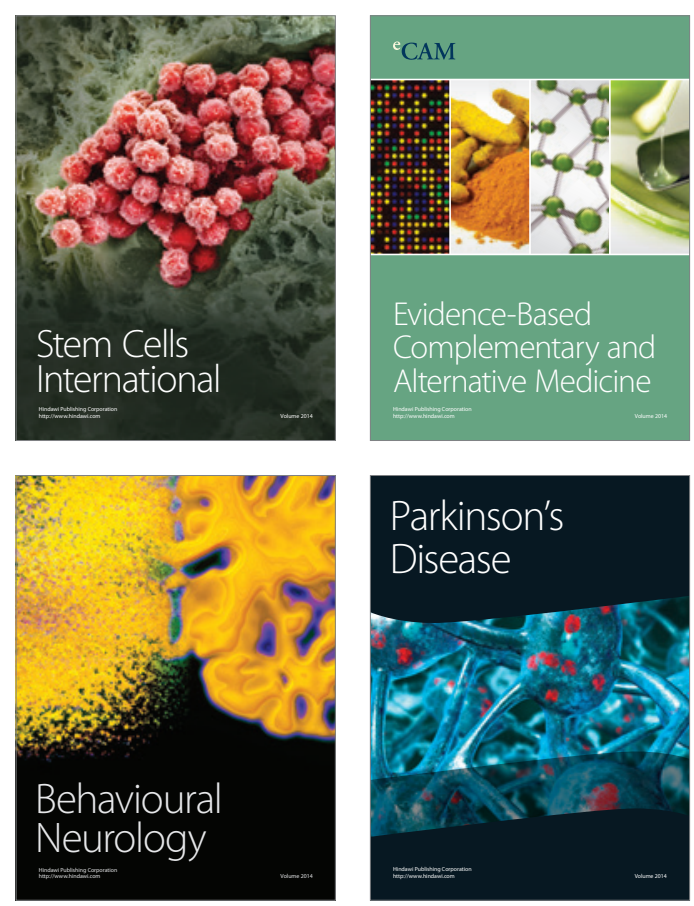

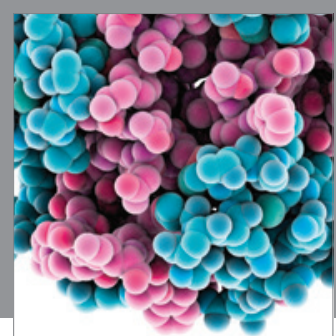

Journal of
Diabetes Research

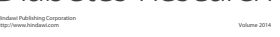

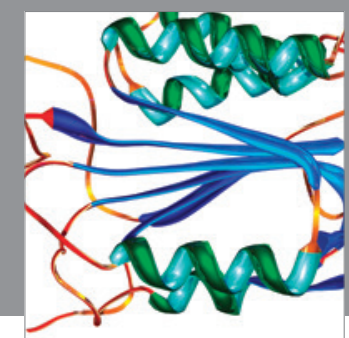

Disease Markers
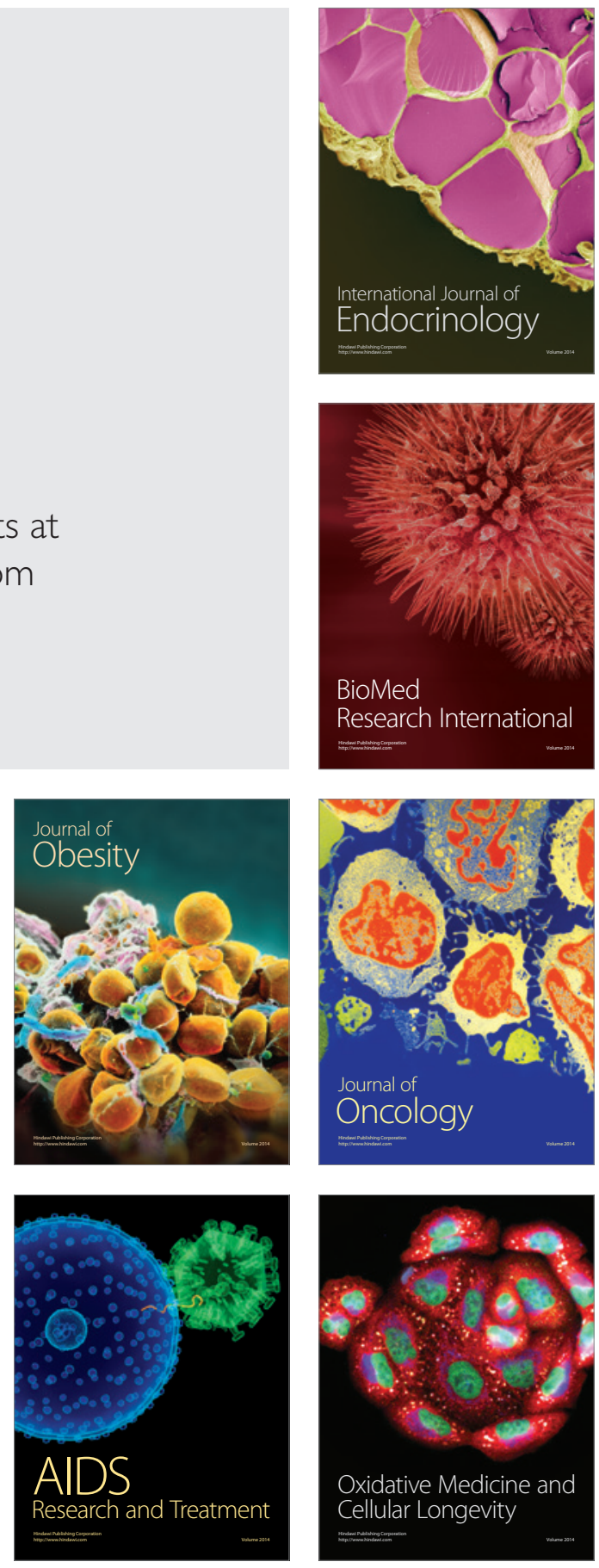